\title{
Analysing Estimators in the Alternative Echo Integrator Method
}

\author{
Magnar Aksland*
}

Department of Biology, University of Bergen, Norway

\begin{abstract}
New and existing estimators of the "Echo Value Constant" based on split-beam detected and integrated single target echoes in the "alternative echo integration method" have been compared by using acoustic data. Two new estimators were made to make consideration to the fact that the detected echo strengths of single targets outside the half power angle of the split beam transducer have positive average bias. Another new estimator that was proposed in a previous paper of the same author is, however, not of this type, and seems in general to be positively biased. The other new estimators seems to be better with respect to the bias than the old one, but one of them seems to be a bit unstable with significant negative bias in special cases. There may be developed even better, nearly beam independent, estimators of the Echo Value Constant.

"In situ" single echo Target Strengths, although not used, are computed, and these indicate that the increasing average positive bias with increasing beam angle is not dramatic within the angle of $-10 \mathrm{~dB}$ transmit beam damping.
\end{abstract}

Keywords: Echo integration in terms of energy, Single target echoes, Position related echo strength bias, Fish density estimation.

\section{INTRODUCTION}

An alternative echo integration method was given in [1]. This is a method that bases the conversion of integrator values to fish abundance on the energies of single target echoes instead of echo intensity (Target Strength). In the alternative method, the conversion factor between "echo abundance", as defined in [1], and fish abundance is based on a collection of representative integrated single target echoes from fish "in situ". The conversion factor is termed the mean Echo Value Constant, and is the average contribution per fish to the echo abundance. A main difference between the alternative echo integration method and the classical echo integration method is that the former does not use single echo peak values, but bases the conversion factor "Echo Value Constant" on integrated single target echoes. This means that the Target Strength concept is not used. Instead, echo strength is expressed by a concept called "backscattering energy" that is defined in terms of the integrated single target echo, or the echo energy. In a symposium on fisheries acoustic in 1982, R. E. Craig proposed to replace power with energy in sonar theory, see [2]. This is exactly what the alternative echo integration method does. Note that the concept "backscattering energy" was defined in [1] and used in [6] under the name "backscattering power". However, backscattering power is not a good name in a model that is based on echo energy, and will hereafter be termed "backscattering energy".

The reason for using integrated single echoes instead of peak values is because the echo integration method integrates the acoustic signal. According to the random phase hypothesis this gives a value that, in average, is equal to the

*Address correspondence to this author at the Department of Biology, University of Bergen, Norway; Tel: +47 55584479; Fax +47 55584450; E-mail: magnar.aksland@bio.uib.no sum of the integrated single echoes in the acoustic signal even if echoes overlap. A requirement is of course that the shadow-effect [3] is negligible. It is demonstrated in Fig. (5) that single echo peak values and the integrator values are rather linearly related.

Two different types of estimators for the mean Echo Value Constant has been developed in [1], but one of these could not be used without modification because it requires representative single target fish echoes at detection angles between zero and an angle with the acoustic axis where the transmit-receive beam function has fallen $-20 \mathrm{~dB}$. It is not possible to collect a representative sample of single fish echoes in this detection angle interval because many echoes far out in the beam are not detected. The purpose of this paper is to investigate an alternative modification of this estimator that compensates for the loss of many single target echoes in the outer part of the main lobe. The echo strengths of single echoes from this region are positively biased in average, mainly because the majority of the echoes that are lost are the weakest.

There is another cause that generates an average positive bias of the beam-corrected echo strength, or backscattering cross-section, of single echoes detected by the split beam system. This is called position related bias in estimates of the acoustic backscattering cross-section, and is caused by random errors in the detected phase angles and the curvature of the beam function. This is explained by Fleischman and Burwen in [4], and has biggest effect on the detected single echoes from far out in the beam. Although beam corrected echo strengths are not used in the alternative echo integration method, errors in the detected phase angles will also cause a bias in the average integrated single target echoes. Target Strengths are shown in some of the figures in this paper, but are not used. 
There are also studies of different factors that influence the "in situ" Target Strength of single fish, see [5]. Although such knowledge may sometimes be useful, the collection of single target echoes to be used in converting echo-integrator values to fish abundance require echoes that are representative for all echoes that are integrated. If this is fulfilled, the factors that might have affected the fish Target Strengths are not of importance.

In this paper, new modifications of both estimator types given in (1) and (8) in [6] are proposed, tried and compared. Special versions of both types of estimators were applied in the same paper. Here we will consider versions and modifications that reduce the effect of the positive bias in average detected backscattering strength of single target echo received at big detection angles in the main lobe.

Every single target echo detection algorithm tests each candidate echo for coming from a single target only. Echo duration, echo shape and stability of the phase angles are investigated and tested before an echo is accepted to be a single target echo. Echoes that do not pass one or more of these tests have other echoes of no-negligible strength to close so that at least the echo tails overlap. Phase angles and echo shapes of weak echoes may also be affected by noise. The echoes that are most likely to be disturbed by other echoes and noise are the weakest echoes. As most of the weakest echoes are those that are received from targets far out in the man lobe, it is natural that the fraction of echoes that do not pass the "single target tests" increases with the detection angle. Also, the fraction of echoes not passing the "single target tests" at a given detection angle are mainly the weakest echoes received at this angle. This is the main cause of the increasing positive bias of average echo strength with increasing detection angle. In [7] a multi-frequency method to decide if echoes come from single targets is described. This method discards a considerable bigger fraction of echoes than that of a single frequency routine.

The acceptance of perfectly overlapping echoes of similar strengths as one single target echo is also a source of positive bias in estimated average echo strength, but this is judged to happen rather seldom in this application both because of low fish density, but also because of the single target echo detection algorithm used here (described in [6]). This routine is, as far as the author knows, the only single target detection routine that integrates single echoes.

Analyses of several single target detection routines including the possibility of accepting multiple echoes as single target echoes have been considered in [8]. Otherwise, many papers on the use of "in situ" single target echoes exist. These are mainly concerned with estimating the mean backscattering cross section of individual fish to be used in the classical echo integration method, and are not fully relevant to the study in this paper that uses integrated single target echoes.

\section{MATERIAL AND METHOD}

Files of raw data recorded by SIMRAD EK 60 scientific echo sounders are used in this study. Recordings from a survey on cod in Lofoten, Northern Norway by the new "G. O. Sars" in 2004 and from a survey on Redfish in the deep Troms $\varnothing$ basin by a hired vessel in 2008 are selected. Most of the cod recordings are from moderate depths (a few hundred meters) where the fish stayed close over the bottom. The redfish recordings, however, were from deep waters (several thousand meters deep) and the recorded fish, although down to $600 \mathrm{~m}$ deep, were very far above the bottom.

The echo integration method in terms of energy described in [1] is used. An application of this method was given in [6], but there the effect of positive biased average echo strengths was not considered.

Estimator type (1) in [6] was modelled by assuming that the average beam dependent backscattering energy (not beam-compensated integrated single target echoes) of fish as a function of observation aspect (observation angle from the acoustic axis) is proportional to the transmit-receive beam function. This assumption is likely to be good for beams where the majority of the single targets are received at angles less than 10 degrees, ([1], Fig. 2). The observation aspect angle is expressed by the symbol $\theta$ in subsequent mathematical formulas.

Only circular symmetric beam functions are considered in this paper. A theoretical model for the beam function was used. This is the Bessel function formula for the beam of a circular piston transducer, see [9]. The model can be expressed by (3) in [6]. i. e.

$$
\mathrm{E}\left[\varsigma_{r b}(\theta)\right]=a\left[B_{s}(k \theta)\right]^{4},
$$

where the left side is the expected beam dependent backscattering energy of fish at observation aspect $\theta$, while $\left[B_{s}(k \theta)\right]^{4}$ is the Bessel function model for the transmitreceive beam function. The value of the parameter $k$ determines the opening angle of the beam while $a$ is the factor of proportion between the average beam dependent backscattering energy of fish and the beam function.

The derivation in [6] leads to the following estimator for the mean Echo Value Constant $\bar{\Psi}$ ((5) in [6]):

$$
\widehat{\bar{\Psi}}=\frac{\hat{a}}{k_{b}{ }^{2}} 1.4406
$$

where $\hat{a}$ is an estimator of $a$. The parameter $k_{b}$ is the particular value of $k$ that gives the theoretical beam the same half power opening angle as that of the used transducer. The modelled beam function is then assumed to be a good enough approximation for the actual beam function in use. The half power opening angle of the used transducer is given in the files of raw data of acoustic recordings. To control this beam model against the measured beam function by SIMRAD of a $38 \mathrm{kHz}$ transducer commonly used on Research Vessels, the measured and modelled beam are plotted in the same coordinate system in Fig. (1). The half power angle for the measured beam is not given, so the fit here is made by trial and error with modelled beams of different values of the parameter $k$. The figure shows that the model is rather good.

$$
\text { Since } B_{s}^{2}(0.8069)=1 / 2, k_{b}=0,8069 / \theta_{1 / 2, r} \text {, where } \theta_{1 / 2, r}
$$
is the half power opening angle in radians from the acoustic axis of the transmit beam.

Inserting this in (2), gives: 


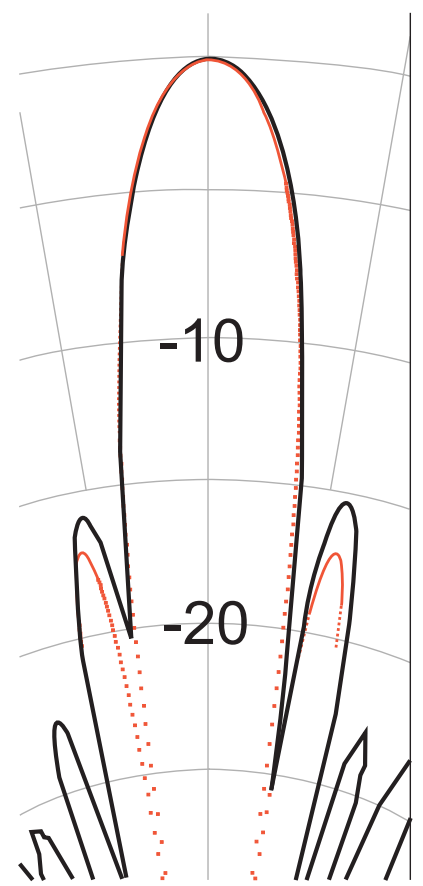

Fig. (1). The measured (black) and the modelled (red) transmit beam function plotted in logarithmic polar coordinates. The fit is absolute good enough in the main lob above the side lobes. The numbers express decibels.

$\overline{\bar{\Psi}}=\frac{\widehat{a}}{\theta_{1 / 2, r}^{2}} 0.9379568$, where $\theta_{1 / 2, r}$ is expressed in radians, and,

$\overline{\bar{\Psi}}=\frac{\widehat{a}}{\theta_{1 / 2, d}^{2}} 3079.13$, where $\theta_{1 / 2, d}$ is expressed in degrees

To use this estimator, it suffice to obtain an estimator of $a$. This is based on a curvilinear regression to the model given by (1) of detected single target echoes with associated phase angles. The least squares method was used. This was also done in [6]. here.

A slightly more general weighted regression is given

Let $w_{1}, \theta_{1}, w_{2}, \theta_{2}, \cdots, w_{n}, \theta_{n}$ be $n$ detected and integrated single target echo-intensities with associated observation angles as in [6]. Let

$$
Q_{v}(a)=\sum_{i=1}^{n} v\left(\theta_{i}\right) s_{i}\left[w_{i}-a B_{s}\left(k \theta_{i}\right)\right]^{2}
$$

be the weighted sum of squared deviations between the observed and expected beam dependent backscattering energies. The function $v(\theta)$ is a general $\theta$-dependent weight function that must be specified by the user, and $s_{i}$ is the sailing speed when the i'th echo is detected. This allows users to choose weight functions with low values at angles where the echo strengths are assumed to be biased or not quite correct. The reason for weighting the terms in (4) by the sailing speed is to correct for skew sampling density of the detected single echoes, because the number of echoes per unit sailed distance is proportional to the inverse of the sailing speed. This weighting is necessary when both the sailing speed and fish density is variable. If the ping repetition rate is variable during the collection of a series of single target echoes, each term in (4) should also be weighted by the inverse of the ping repetition rate, but this was not needed here. In order to avoid three multiplicative weights in (4), $s_{i}$ may designate the sailed distance between successive pings at the detection times of each echo. The least squares estimator of $a$ is that function that minimizes $Q_{v}$, and is obtained by equating $\partial Q_{v} / \partial a$ to zero. This gives

$$
\widehat{a}=\frac{\sum_{i=1}^{n} v\left(\theta_{i}\right) s_{i} w_{i} B_{s}^{4}\left(k \theta_{i}\right)}{\sum_{i=1}^{n} v\left(\theta_{i}\right) s_{i} B_{s}^{8}\left(k \theta_{i}\right)}
$$

In [6], the weight function $v(\theta)=\theta^{2}$ was argued for and used. However, if the $w_{i}$ 's have, on average, positive bias for big $\theta$, it could be better to choose a weight function that reduces the influence of echoes with big values of $\theta$ more than in [6]. All $\theta$-dependent weight functions tried here are zero when $\theta>\theta_{-20}$, where $\theta_{-20}$ is the angle where the transmit-receive beam has dropped $20 \mathrm{~dB}$. That is, single target echoes with detected off axis angles bigger than $\theta_{-20}$ are not used for estimation purposes, although they are shown in some of the figures. The following two weight functions in addition to that used in [6] are used, and the corresponding estimates of the mean Echo Value Constant are compared:

1: $\quad v_{1}(\theta)=1$ and

2: $v_{2}(\theta)=1-0.9\left[\frac{\theta-\theta_{1 / 2}}{\theta_{-20}-\theta_{1 / 2}}\right]^{2} \quad$ when $\theta_{1 / 2} \leq \theta \leq \theta_{-20}$, and 1 when $\theta<\theta_{1 / 2}$

The weight function $v_{2}$ reduces the influence of echoes received at angles bigger than the half power angle relative to that of $v_{1}$.

The other type of estimator for the mean Echo Value Constant used here is derived in [1] and is given by (8) in [6].

$$
\hat{\Psi}_{3}=\pi \tan ^{2} \theta_{c} \frac{1}{n} \sum_{1}^{n} w_{i} \cos ^{2} \theta_{i}
$$

The parameter $\theta_{c}$ in (6) is a large angle in the main lobe that limits the angle interval from which single target echoes are used for estimation purpose. Arguments in [1] conclude that $\theta_{c}$ can be set equal to $\theta_{-20}$, but not smaller as this will cause a significant negative bias in the estimate. The value $\theta_{-20}$ will be used for $\theta_{c}$ in applications in this paper. An advantage with this estimator is that it does not need any model for the average beam dependent backscattering energy of fish. However, it requires a representative sample of sin- 
gle target echoes from the observation angle interval between zero and $\theta_{-20}$. Usually it is not possible to obtain a sample of single echoes that follows the theoretical distribution (9) in [6] of the number of echoes at different values of the observation aspect. Most observed samples seem to lack a considerable number of weak echoes, in particular at angles that approach $\theta_{-20}$. There are different ways to modify (6) to reduce the effect of too few echoes at big aspect angles. In [6], two different modifications were proposed, and one of these was tried and seemed to work well. The other proposed modification is tried in this paper. This is to replace the sum in (6) by a weighted sum where each term is multiplied by a weight that is the ratio of the expected and observed number of echoes in each of a set of narrow intervals for the observation aspect angle. One may ask about the point in trying to modify (6) when estimator type (5) may be used. This is because a successful modification of (6) may be used without a calibrated echo sounder and beam function, although it does require a value of $\theta_{-20}$ to be known approximately.

The theoretical probability density for the observational aspect is derived in [1], and is given by

$$
f\left(\theta \mid \Theta \leq \theta_{c}\right)=\frac{2}{\tan ^{2} \theta_{c}} \frac{\tan \theta}{\cos ^{2} \theta} \text {, for } 0 \leq \theta \leq \theta_{c}
$$

This density holds under the hypothesis of uniformly distributed fish positions in a local horizontal plane, and that the echoes from all fish at observation aspect less than $\theta_{c}$ are detected. The graph of this density for small angles is close to a straight line with positive slope through the origin. In the interval from 0 to $\theta_{-20}$ for most transducers used in fisheries acoustic, the approximation

$$
f\left(\theta \mid \Theta \leq \theta_{c}\right) \approx b \theta
$$

for some constant $b$, is good enough. As long as the histogram of the observation aspect angles of detected single target echoes grows approximately linear outward from the acoustic axis, the detected echoes seems to follow the distribution (8). However, most empirical histograms of the observation aspect grow linearly out to around the half power opening angle after which it bends down, indicating that there are targets here that have not been detected as single target echoes. See Fig. (2).

To modify the estimator (6) against the effect of lost single target echoes mainly far out in the beam, the detection angle interval is divided into subintervals having equal probability as determined from (8). By integrating (8) from $\theta_{i}$ to $\theta_{i+1}$, it may be shown that the $n$ subintervals made up by

$$
0<\theta_{1}<\theta_{2}<\cdots<\theta_{n}
$$

have equal probabilities when

$$
\theta_{i}=\sqrt{\frac{i}{n}} \theta_{n}, \quad i=1,2, \cdots, n
$$

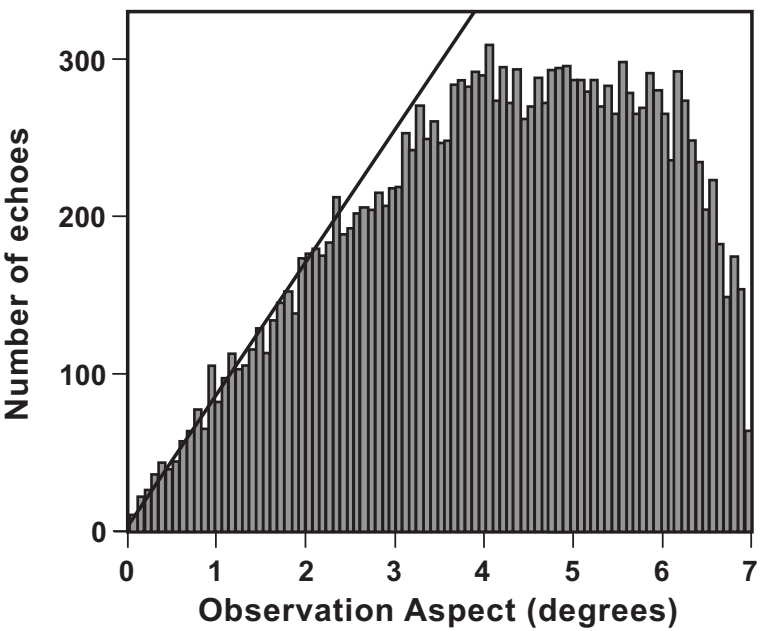

Fig. (2). Empirical distribution of observation aspects from 23000 fish targets. We see that this distribution holds well relative to (8) out to slightly more than 2 degrees, and fairly well out to more than 4 degrees. The used transducer had a half power angle of $3.54 \mathrm{de}-$ grees, and an angle at $-10 \mathrm{~dB}(-20 \mathrm{~dB}$ transmit and receive) damping at 6.05 degrees.

A programme divides the observation aspect angle interval up to $\theta_{-20}$ into 100 subintervals satisfying (9) if the number of detected single targets is 1000 or bigger. If the number of echoes is between 100 and 1000 the number of subintervals is one 10-th of the number of echoes. The programme does nothing if the number of echoes is less than 100. The programme counts the number of echoes with observation aspect in each subinterval and compute weights $v$ that are the inverse of these numbers. The modified version of (6) is then computed as

$$
\widehat{\Psi}_{3 m}=\pi \tan ^{2} \theta_{c} \frac{\sum_{1}^{n} w_{i} v_{i} s_{i} \cos ^{2} \theta_{i}}{\sum_{1}^{n} v_{i} s_{i}},
$$

where $v_{i}$ has the same value for each echo with observation aspect in the same subinterval. The sailing speeds, or sailed distance between successive pings, $s_{i}$ are also used by the same reason as in (5).

As each subinterval has the same value for the expected number of echoes, the intervals with the lowest number of echoes get the highest weights. Although echoes with phase angles in subintervals with few echoes are weighted up in (10), there is still a reason for (10) to be positively biased. The echoes from far out in the beam that are fewer than predicted by (8) are likely not to have the same distribution of echo strengths as echoes from targets closer to the acoustic axis. This is because echoes that fail to be detected are expected to be the weakest. The detected echoes will therefore have higher average echo strength than it would if no echoes had been lost. For this reason a third modification of (6) is proposed.

Instead of weighting up echoes in aspect subintervals with few echoes, we add a number of artificial echoes with 
zero echo strength into subintervals with few echoes relative to the average number of echoes in the subintervals closest to the acoustic axis. To compute this estimator, the subintervals for which the average number of echoes is to be calculated must be specified. It is natural to choose the subintervals with upper limit less than a given angle. This angle should have a value close to an angle where the histogram of detected phase angles starts to fall off relative to (8). Although this is variable, we have here used slightly more than $60 \%$ of the half power angle, which is an angle where (8) are fulfilled in most applications. All subintervals further out with fewer than the computed average number of echoes is added artificial echoes with zero echo strength until the average number of echoes is reached. A formula for this estimator may be written as

$\hat{\Psi}_{3}=\pi \tan ^{2} \theta_{c} \frac{1}{\sum_{i=1}^{n} s_{i}+\sum_{j=1}^{m} s_{j}} \sum_{i=1}^{n} w_{i} s_{i} \cos ^{2} \theta_{i}$

where $m$ is the total number of artificial echoes that are added. Note that these echoes do not contribute to the value of the rightmost sum in (11). The sailing speeds corresponding to the added echoes have to be included in the sum of sailing speeds in (11). These are not defined and have to be stipulated. This is a delicate problem because the different single target echoes in a particular observation angle subinterval comes from different parts of the surveyed cruise line that may have different sailing speeds. It should be the sailing speeds corresponding to the single target echoes that failed to be detected, and these are not known.

This case, as well as different reasons causing nonrepresentative single target echoes, is taken up in the discussion section.

Under the assumption that the sailing speeds of the undetected single target echoes have the same average speed as that of the detected echoes, we may write:

$\widehat{\Psi}_{3}=\frac{\pi \tan ^{2} \theta_{c}}{\frac{n+m}{n} \sum_{i=1}^{n} s_{i}} \sum_{i=1}^{n} w_{i} s_{i} \cos ^{2} \theta_{i}$

Estimator (12) is used in this analysis. Since the echoes that are not detected, although weak, are definitely bigger than zero, the estimator (12) is expected to have negative bias. However, to get an idea whether the bias of (10) and (12) are significant, computations of the estimators from field data are necessary. This is the purpose of the next section.

\section{RESULTS}

The results are presented by tables and a set of standard graphs for the different selected files of acoustic raw data. The graphs show the Echo Values corresponding to (10) and (12) which are the graphs of the estimators as functions of a variable angle $\theta$ substituted for $\theta_{c}$. Note that the Echo Value Constant is the value of the near constant level of the Echo Value, that is the Echo Value when $\theta \geq \theta_{-20}$.
A program computes the estimators for 20 equally spaced values for $\theta$ between zero and $1.15 \theta_{-20}$. These functions are plotted together with a scaled Echo Value of a sphere ([1], Fig. 1) computed from the model of the beam function. This is done to study and compare the shapes of the different Echo Values. In addition, the distribution of the peak values of the single target echoes used to compute the Echo Value functions are shown graphically. A threshold has removed weak echoes from plankton and other small organisms in this plot. Finally the histogram distribution of the observation aspect angles corresponding to the same echoes is shown.

An echogram from the acoustic recording is shown first, together with different plots of target strength distributions of detected single target echoes.

Different values associated with the run of each selected file of raw data are given in tables. These tables include estimates of fish density which is the ratio between the Integrator Value and the Echo Value Constant. To reduce the influence by noise as well as echoes from plankton and other smaller organisms not belonging to the target population, suitable thresholds are used. Here one integrator threshold is used when integrating the $20 \log \mathrm{R}$ TVG signal to compute the Integrator Value, while another single echo threshold is used to filter away single target echoes from alien organisms before computing the Echo Value Constant.

A value is set for the single echo threshold by studying plots analogous to the Target Strength against Observation Aspect and Echo Depth. This threshold is difficult to set when the Target Strength of fish and plankton overlap. However, the estimates of the Echo Value Constant are sensitive for this threshold, in particular when many echoes have values close to the threshold.

A value for the Integrator threshold is easier to set since the Integrator Value is often not very sensitive to this threshold. See how the Integrator value varies with the Integrator threshold in [6]. A plot of the peak values against the observation aspects of all single echoes is used, as shown in Fig. (3).

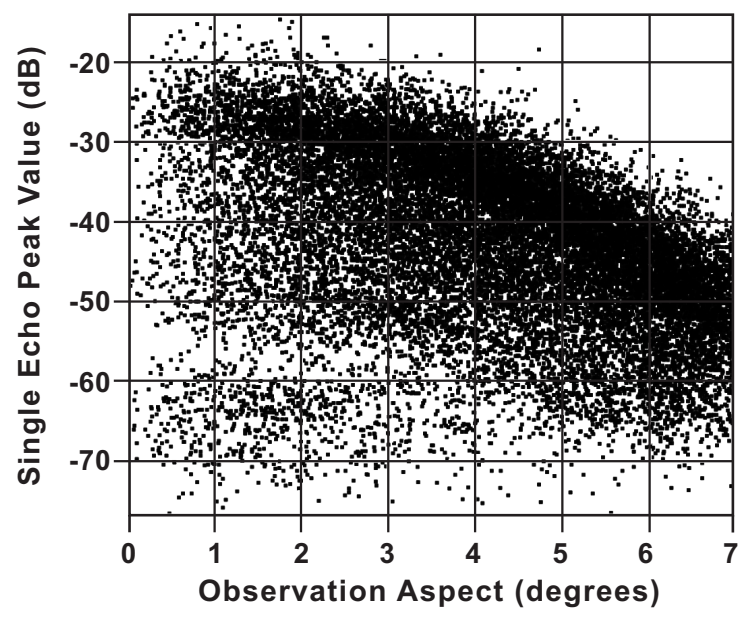

Fig. (3). Peak Values against Observation Aspects.

The transducer here is the same as in Fig. (2). 
Based on Fig. (3), it is natural to choose a value of -60 $\mathrm{dB}$ for the Integrator Threshold. Then, most of the plankton echoes, that are in the cloud below the fish echoes, are below the threshold. However, some of the weakest fish echoes from far out in the beam are also below the threshold. Setting of an Integrator threshold is always a compromise between accepting some plankton echoes close to the acoustic axis while sacrificing some fish echoes far out in the beam. The threshold set here is for the $40 \log \mathrm{R}$ TVG signal, but it is applied to the $20 \log R$ TVG signal in the sense that this is integrated only when the $40 \operatorname{logR}$ TVG signal is above the threshold. For the different files analysed in this section, the Integrator threshold is set based on figures like Fig. (3), but where the unit for the peak values is in accordance with the echo signal level used. Bias caused by the Integrator threshold is judged to be small in the applications in this paper based on experience from [6]. Model calculated thresholdinduced bias in echo integration of single echoes have been studied in [10] and [11], but their results are not used in this paper because selection of threshold values are based on selected figures as explained.

The first three files of acoustic raw data analysed are from Lofoten, Northern Norway in March/April 2004. Although these recordings are from 5 different operating frequencies and transducers, only $38 \mathrm{kHz}$ data are studied here. The corresponding transducer had an average half power opening angle of 3.54 degrees, and an angle of $-20 \mathrm{~dB}$ transmit-receive intensity of 6.05 degrees. For all the selected recordings analysed in this paper the pulse length was $1 \mathrm{~ms}$, and the echo signal was sampled each $0.256 \mathrm{~ms}$ giving roughly 4-6 samples over one echo pulse.

The first file is from a recording west of Lofoten 24 March, 2004 at night, and results are shown in Figs. (4 and 5), and in Table $\mathbf{1 .}$

Although beam compensations are computed from the beam model, which may be slightly narrower far out in the main lobe than the used beam, Fig. (4) demonstrates clearly the effect of positive biased average target strength with increasing observation aspect. The Target Strength against Depth plot shows how weak and strong echoes are distributed with depth. Similar plots, where Target Strength is replaced with the beam compensated integrated echo intensity, are used to determine a value of the single target threshold. These plots are very similar to the plots in Fig. (4), since the integrated single target echo intensities are very close to be- ing linearly related to the corresponding peak values as shown in Fig. (5).

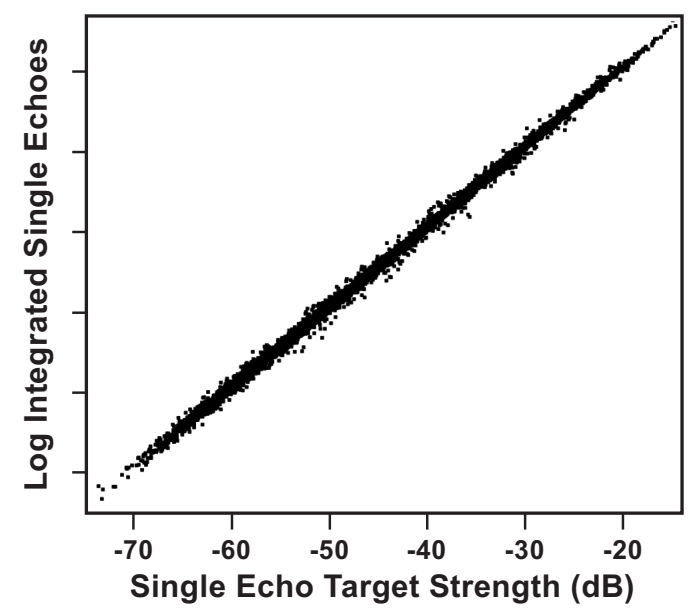

Fig. (5). Relation between Target Strengths and corresponding beam compensated Integrated Echo Intensities for the single echoes used in Fig. (4).

Echoes weaker than around $-50 \mathrm{~dB}$ are here judged to come from plankton and will be removed by the single target threshold.

The Echo Value Constant is the value of the Echo Value function at the angle $\theta_{-20}=6.05$ degrees. The different estimates are given in Table $\mathbf{1}$, as well as corresponding estimates in subsequent tables. Note that an echo signal level different from the standard has been used during these calculations. This level affects the sizes of Integrator values and Echo Value Constants, but not the estimates of fish densities. The integrator value is the sailing speed weighted average integrator value per ping, and the corresponding estimated fish densities are the average for the sailed distance. Estimated fish densities corresponding to the Echo Value Constants from (10) and (12) are not given in the tables because these Echo Value Constants are computed to be compared with the other Echo Value Constants and are not assumed to be quite correct.

The tables also give three values for mean Target Strength. Although the alternative echo integration method does not use Target Strength, this is done to show the effect of all sources of position related bias of Target Strength. The
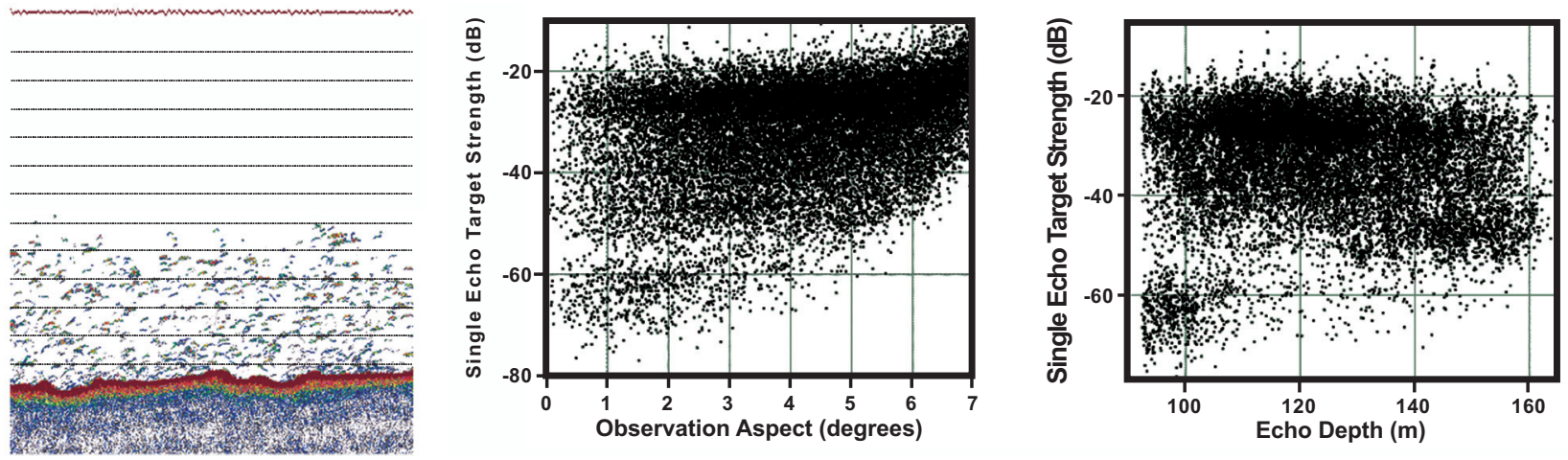

Fig. (4). Echogram with depth division lines for each $12.5 \mathrm{~m}$ depth, distribution of all detected single target echoes of Target Strengths against observation aspects, as well as all echoes with observation aspect less than $\theta_{-20}$ of Target Strengths against echo depths. 

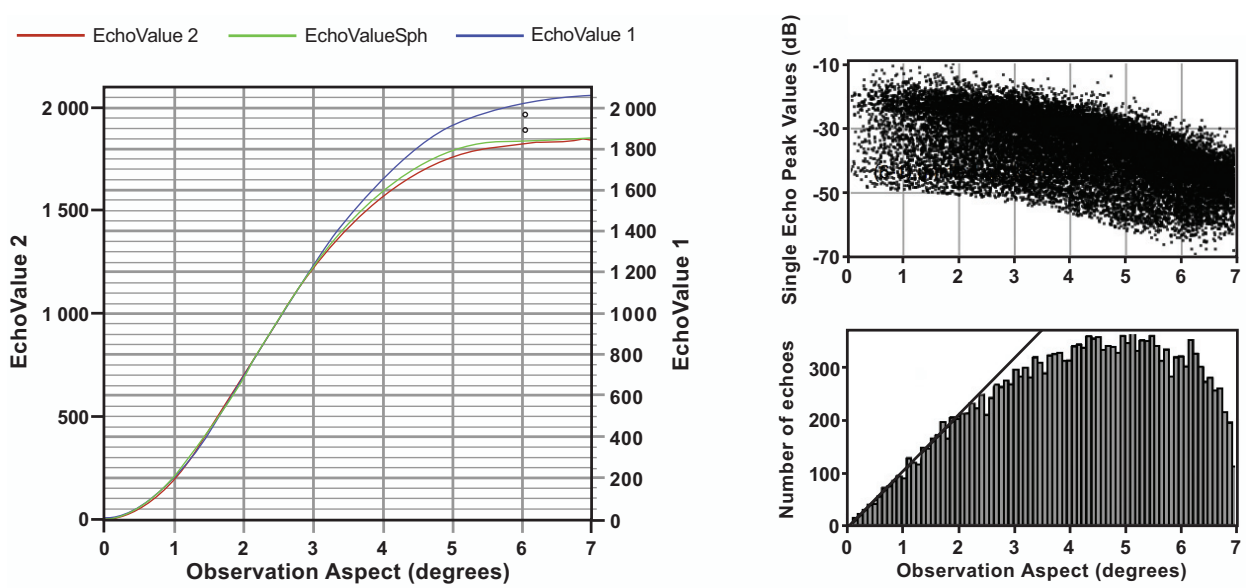

Fig. (6). Left: EchoValue 1 and 2 (as given by (10) and (12) together with the Echo Value of a sphere scaled to be concurrent with the other two at moderate angles. Also, the estimates given by (3) and (5) are shown at $\theta_{-20}$ by the small circles corresponding to different weight functions in (5). Right: Peak values in $\mathrm{dB}$ of all single target echoes at aspect less than seven degrees after a threshold have removed weak echoes assumed to come from plankton and individuals other than the target population. The distribution of observation aspect angles of the same echoes is also shown.

\section{Table 1.}

Number of echoes is 22186 before, and 16622 after applying the single target threshold filter

Sailed distance: $4568 \mathrm{~m}$. Integrator value: 41.973

Echo Value Aksland 2006 Weight function 1 Weight function 2 (10) (12)

$\begin{array}{llll}1969.50 & 1895.48 & 1893.92 & 2005 \quad 1820\end{array}$

Fish density (individuals

pr. square meters): $\quad 0.02131 \quad 0.02214 \quad 0.02216$

Mean TS (see explanation above) $\quad-26.186 \quad-26.952-25.049$
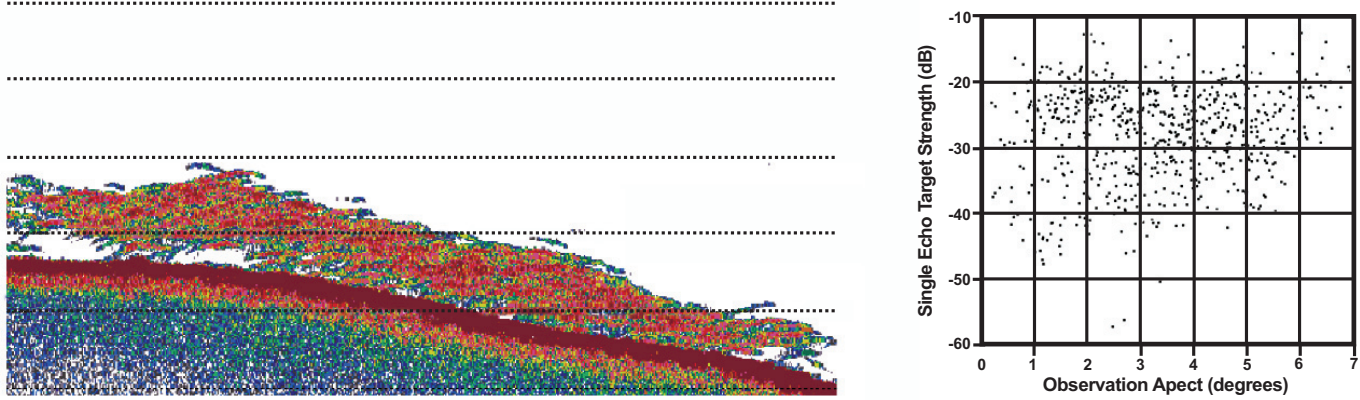

Fig. (7). Echogram (separation between the depth lines is $12.5 \mathrm{~m}$ ) and distribution of Target Strengths against observation aspect.

given Target Strength values correspond to three different average scattering cross sections of the detected single target echoes after using the single target threshold filter. The first number is the simple average for the echoes received at all angles out to $\theta_{-20}$. The second number is the corresponding weighted average where $\theta$-dependent weights proportional to the transmit-receive beam function are used. Then, echoes from close to the acoustic axis have the biggest influence. The last number is the simple average corresponding to the echoes received at angles between $0.8 \theta_{-20}$ and $\theta_{-20}$, that is, the echoes received at the biggest angles. These numbers are computed to demonstrate the effect of positive biased average Target Strengths.

If there are no angle dependent bias, these three average values will be the same. However, if the positive bias in- creases with angle, the first and third value will be to big with the third bigger than the first, and the second will be the smallest and closest to the true value.

The Echo Values in Table 1 using weight functions 1 and 2 in (5) are so close that they cannot be seen as two circles in Fig. (6).

Next, a daylight recording on March 25, 2004 along a dense cod concentration at $110-130 \mathrm{~m}$ depth is analysed. The location is west of Lofoten. Results are shown in Figs. (7 and 8), and in Table 2.

The number of echoes here are small compared to the previous files mainly because of a shorter distance sailed, but also because a smaller fraction of detected single target echoes. The single echo threshold used corresponds to around $47 \mathrm{~dB}$. 

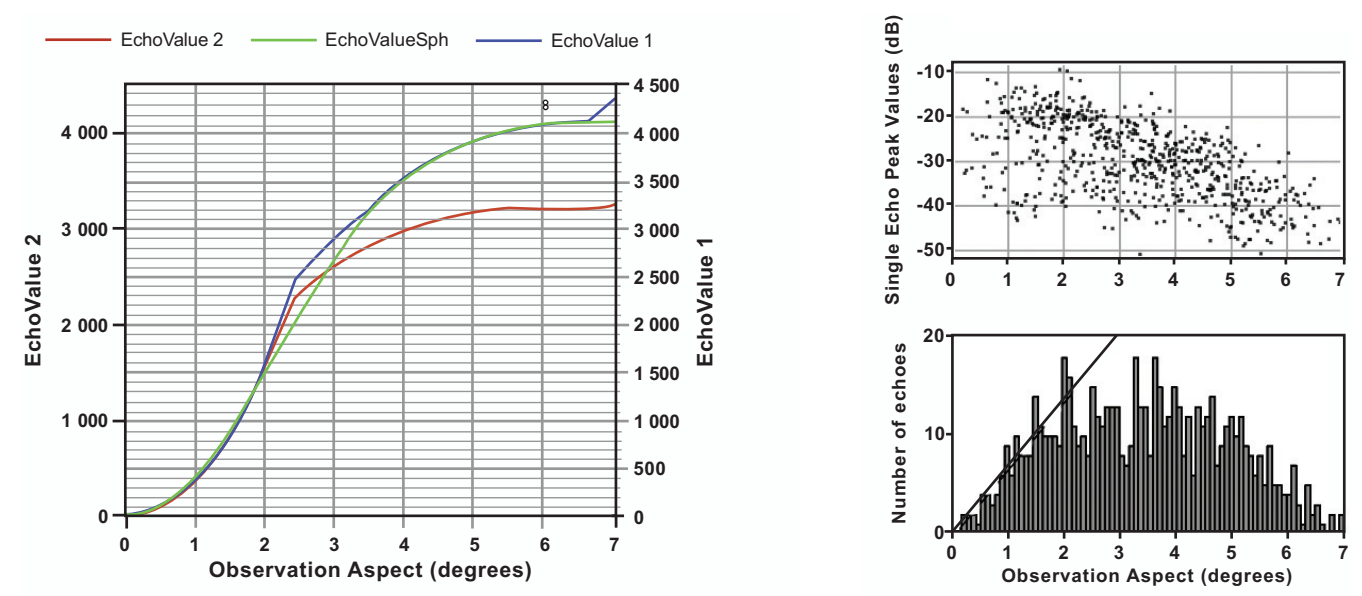

Fig. (8). Echo Values, Peak Values and Number of echoes against observation aspect.

\section{Table 2.}

Number of echoes is 699 before, and 649 after applying the single target threshold

Sailed distance: $629 \mathrm{~m}$. Integrator value: 263.050

Echo Value

Aksland 2006

Weight function 1 Weight function 2

(10)

(12)

4287.35

4338.90

4339.76

$4090 \quad 3202$

Fish density (individuals

$\begin{array}{llll}\text { pr. square meters): } & 0.06135 & 0.06063 & 0.06061\end{array}$

Mean TS (see explanation above Table 1.) -23.895 -23.697 -23.563
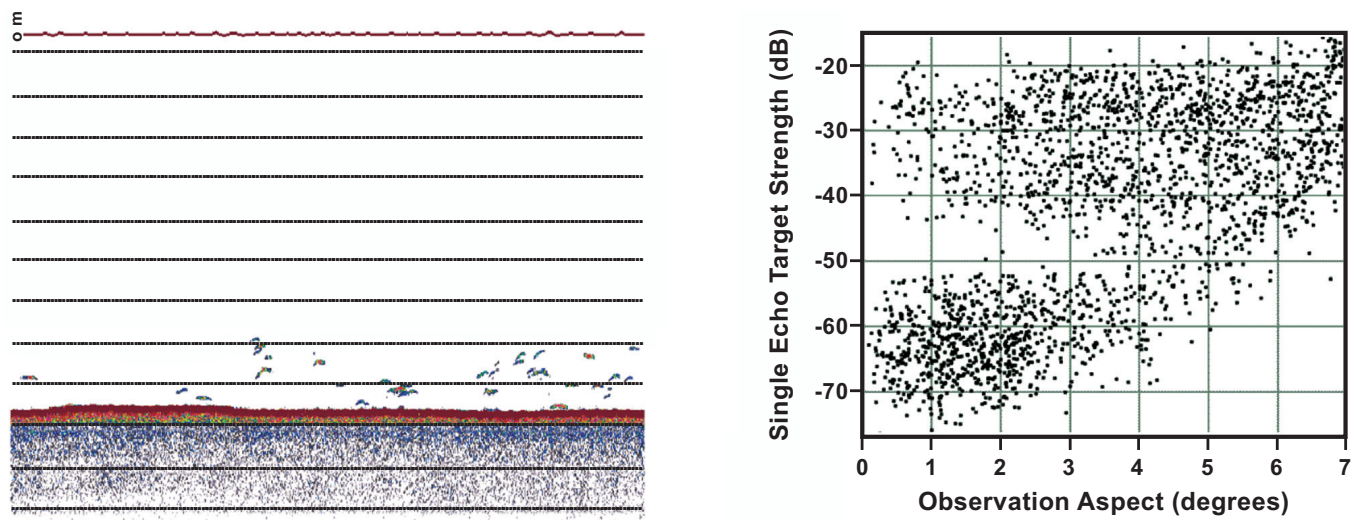

Fig. (9). Echogram (10 m separation between depth lines) and Target Strengths against observation aspect.

The empirical Echo Value functions in Fig. (8) have strange shapes. This may be caused by single echoes not detected, but also because of the low total number of detected echoes. The fraction of detected single echoes here is expected to be small, and these echoes may not be quite representative for all echoes.

Lastly from this cruise, we analyze a recording of cod close to the bottom with results shown in Figs. (9 and 10, and in Table 3).

This is a recording of low fish density. The single echo threshold used corresponds to $-48 \mathrm{~dB}$.

The shapes of the empirical Echo Value functions are not ideal here compared to the Echo Value of the sphere. This happens in cases where the empirical distribution of observation aspects has clear deviations from a straight line outwards from the acoustic axis, as shown in Fig. (10).

At last we will analyze a recording of fish in deep waters. The file is from the deep Troms $\varnothing$ basin in the Norwegian Sea with bottom depths down to $3000 \mathrm{~m}$. Fish density is rather low and single target echoes are detected down to $600 \mathrm{~m}$. The recording is done during late evening September 6, 2007. The transducer used here had a half power angle of 3.52 degrees, and an angle of $-20 \mathrm{~dB}$ transmit-receive damping of 6.02 degrees. The other parameters were similar to that for the 2004 survey analysed above.

Results from the selected file is shown in Fig. (11). 

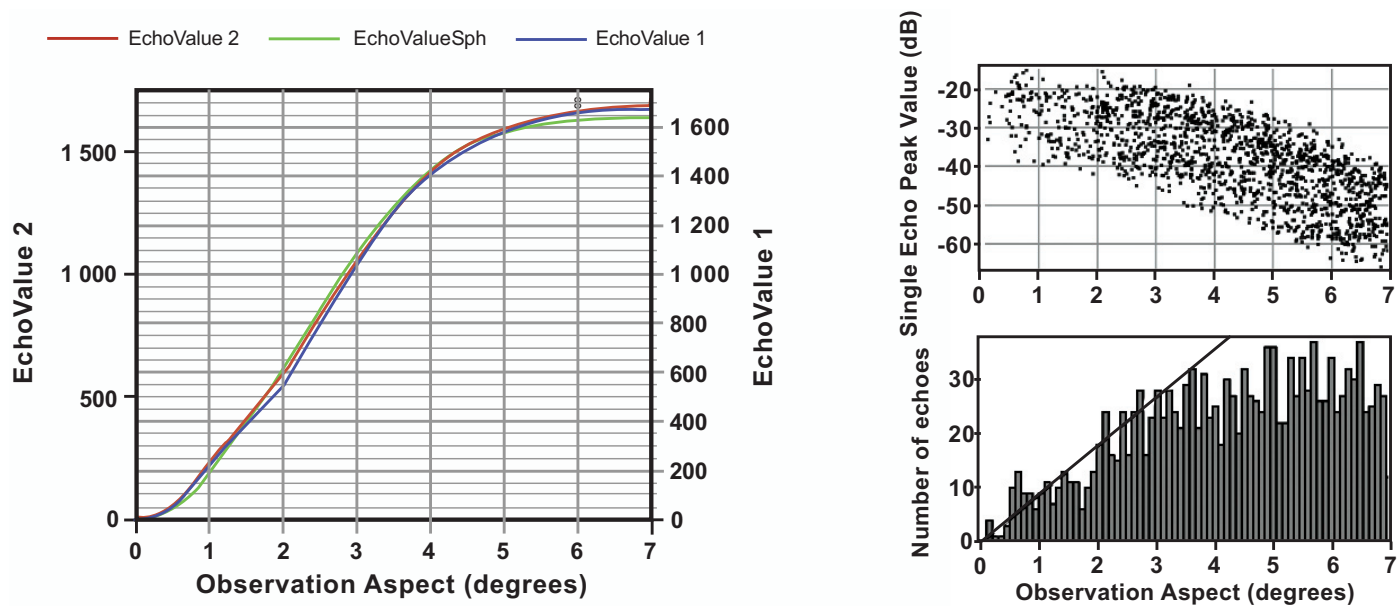

Fig. (10). Echo Values, Peak Values and Number of echoes against observation aspect.

Table 3.

Number of echoes is 1531 before, and 1218 after applying the single target threshold

Sailed distance: $3268 \mathrm{~m}$. Integrator value: 2.916

Echo Value Aksland 2006 Weight function 1 Weight function 2 (10) (12)

$\begin{array}{lllll}1707.74 & 1689.80 & 1689.47 & 1661 & 1660\end{array}$

Fish density (individuals

$\begin{array}{llll}\text { pr. square meters): } & 0.00171 & 0.0173 & 0.00173\end{array}$

Mean TS (see explanation above Table 1) $\quad-27.279-27.574 \quad-26.946$
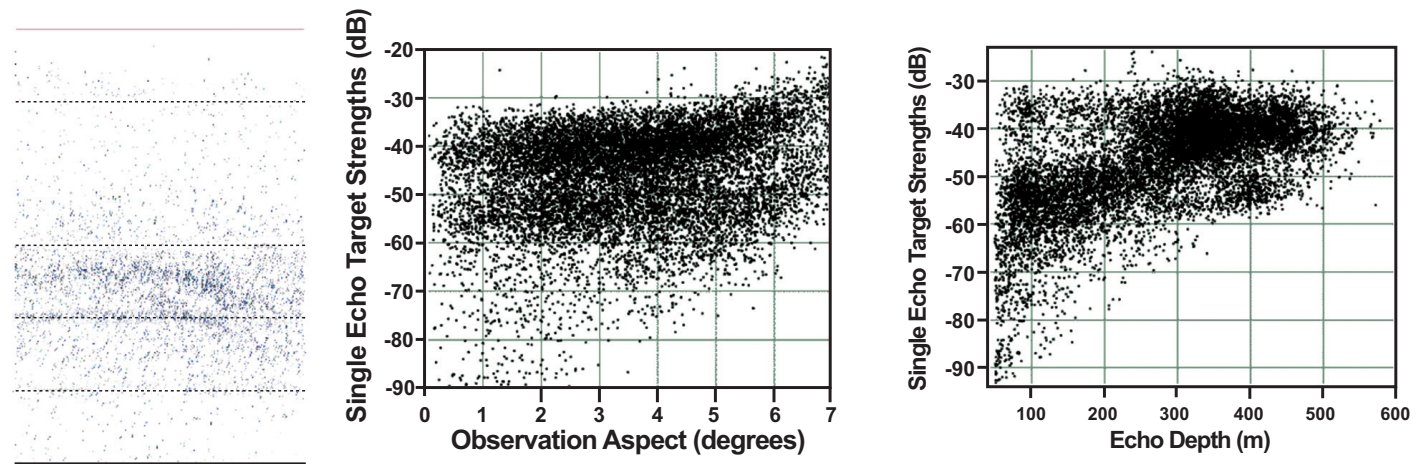

Fig. (11). Echogram (from surface to $600 \mathrm{~m}$ depth), Target Strengths against observation aspects, and against echo depths.

Based on the Target Strength against depth plot in Fig. (11), this file is analysed independently between 50 and 240 $\mathrm{m}$, and between 240 and $570 \mathrm{~m}$ depth. The single echo thresholds are not easy to set, but are decided equivalent to $43 \mathrm{~dB}$ in the shallow, and $-48 \mathrm{~dB}$ in the deep interval.

Due to the big depth range in the recordings of the raw data files from this survey, an analysis of the noise level against depth of the $40 \log \mathrm{R}$ TVG echo signal is performed. The logarithmic signal strength in terms of squared voltage, here called Signal Intensity, from around 200, 400 and 600 $\mathrm{m}$ depths are shown as histograms on a decibel scale in Fig. (12).

The redfish echoes have peak values above $-50 \mathrm{~dB}$ on the acoustic axis. This means that they are above the noise down to $600 \mathrm{~m}$ close to the acoustic axis. Due to the negligible fish density in the deep, the noise will hardly lead to significant bias in the estimates if the Integrator threshold is set above the noise level in the $20 \log \mathrm{R}$ TVG signal. This is much lower than the $40 \log \mathrm{R}$ TVG noise in the deep.

Fig. (13) and Table 4 present the results from the shallow interval (50-240 m).

It was difficult to fit the Echo Value of a sphere to the two empirical Echo Values here because of their anomalous shapes. There are a few very high outliers in the plot of Single Echo Peak Values. These may have biased the estimates. Such peaks may be caused by transmit pulses from other acoustic equipment, for example during communication with trawl sensors. Such peaks may easily be filtered away, but 


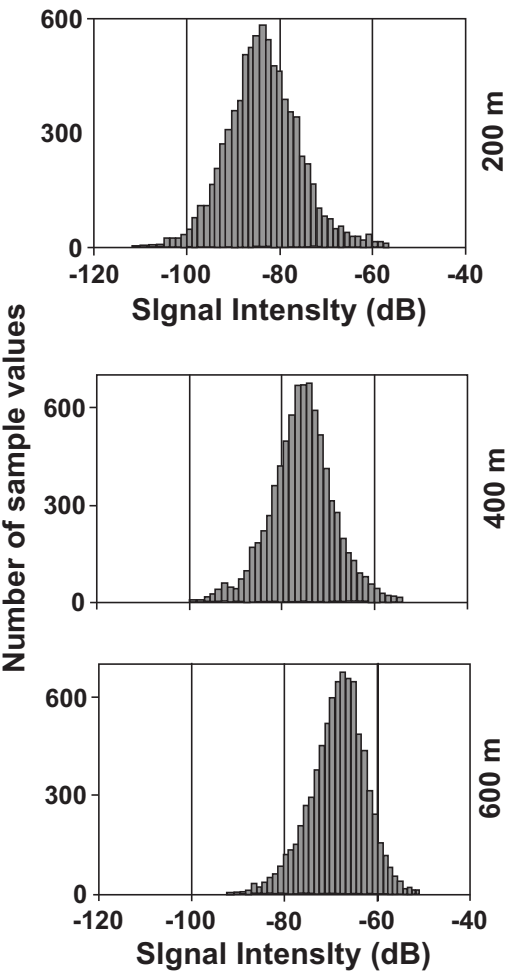

Fig. (12). Noise histograms of the $40 \log R$ TVG signal in two, four and six hundred $m$ depth.

this was not done, as it does not reduce the value of the results of this study.

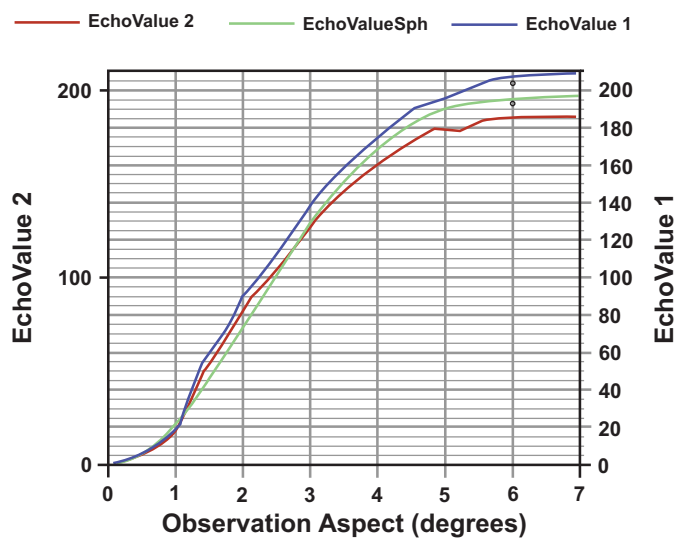

Fig. (14) and Table 5 present the results from the deep interval (240-579 m).

The striking difference between the Figs. (13 and 14) are the observation aspect histograms. It shows that single echoes are lost from 2.5 degrees in the deep interval and from 6 degrees in the shallow interval.

The corresponding fish density per square meter estimates with using only one depth interval (single echo threshold corresponding to $-45 \mathrm{~dB}$ ) are: 0.014410 .001516 and 0.01518 . These estimates are higher, but less than $10 \%$ higher than the corresponding estimates summed over two depth intervals.

\section{DISCUSSION}

The two modified estimators (10) and (12) for the Echo Value Constant both seem to have moderate but significant biases, usually positive for (10) and negative for (12) as expected. As the correct value is not known, it is not possible to specify the exact biases in the different cases in this paper. However, there are reasons to believe that the fitted Echo Value of a sphere in most cases converge to a value that is closer to the true Echo Value Constant than the two modified estimates, in particular when all three Echo Value curves are close to each other for small angles. The bias in the Echo Value functions corresponding to (10) and (12) happens mainly when the observation aspect approaches $\theta_{-20}$, which is slightly more than 6 degrees in the applications in this paper.

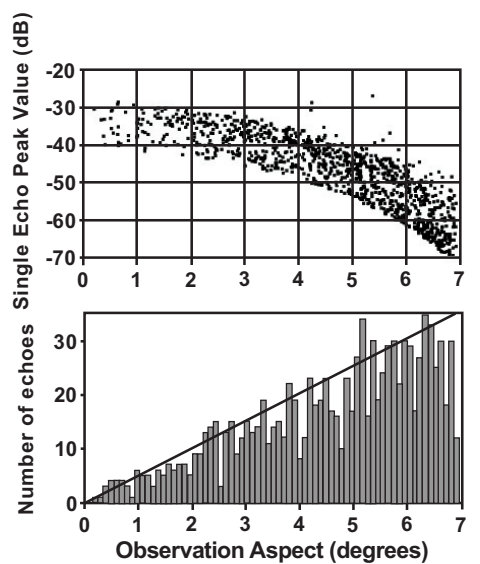

Fig. (13). Echo Values, Peak Values and number of echoes against observation aspect.

Table 4.

Number of echoes is 4886 before, and 1013 after applying the single target threshold

Sailed distance: $9292 \mathrm{~m}$. Integrator value: 0.864

Echo Value Constant Aksland $2006 \quad$ Weight function 1 Weight function $2 \quad$ (10) (12)

$\begin{array}{lllll}192.96 & 198.89 & 198.72 & 207 & 185\end{array}$

Fish density (individuals

$\begin{array}{llll}\text { pr. square meters): } & 0.00448 & 0.00435 & 0.00435\end{array}$ 

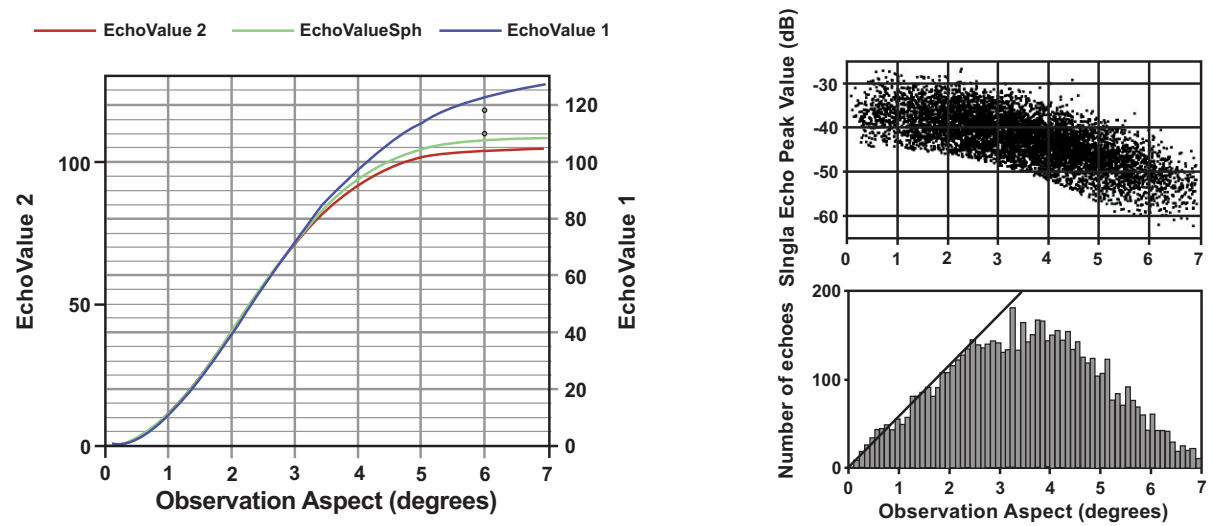

Fig. (14). Echo Values, Peak Values and Number of echoes against observation aspect.

Table 5.

Number of echoes is 7695 before, and 6133 after applying the single target threshold

Sailed distance: $9292 \mathrm{~m}$. Integrator value: 1.060

Echo Value

Aksland 2006 Weight function 1 Weight function 2

(10) (12)

$117.32 \quad 110.39$

110.29

123

104

Fish density (individuals

$\begin{array}{lll}\text { pr. square meters): } \quad 0.00904 & 0.009600 .00961\end{array}$

Sum, both intervals $\underline{\underline{0.01352}} \quad \underline{0.01395} \underline{0.01396}$

Mean TS (see explanation above Table 1) $\quad-38.272 \quad-39.319-35.784$

A lot more files of raw data than those presented in this paper have been analyzed, and corresponding figures have been made. This discussion is therefore based on more experience than that represented by the figures shown here.

The estimates based on (3) seem usually to be closer together than those based on (10) and (12). Without exception the estimates based on Weight function 1 and 2 in (5) are so close that they are difficult to distinguish on the graphs. The corresponding estimates of fish density are also always so close that it may be concluded that the most complicated Weight function 2 is not necessary.

Also, the estimates based on the beam model usually have values between the Echo Value Constants following from (10) and (12) except for difficult cases. It is also striking that the estimate based on Weight function 1 usually are close to the curve of the fitted Echo Value of a sphere. When the three Echo Value curves are close to each other near the acoustic axis, but differ when they pass $\theta_{-20}$, it is likely that the fitted Echo Value of the sphere makes out the best approximation to the true Echo Value function. This indicates that the estimator based on Weight function 1 is rather good, and better than the Weight function used in [6]. Due to the tendency of too high average single echo strength of echoes received far out in the main lobe, the beam model estimator used in [6] is likely to give a slightly overestimated estimate of the Echo Value Constant, and hence a slightly underestimated fish density estimate.

The estimators based on (3) depend on the beam model. Although this seems good (Fig. 1), the best had been to base the estimators on the actual beam function of the used transducer. This is possible if the beam function is precisely known out to the angle $\theta_{-20}$. If the calibrated beam is a fitted model, this could replaced the beam model in (1) if the beam is circular symmetric. If not, the beam depends on two angles, and this is a straightforward generalisation of (1) since the split beam system detects two angles corresponding to each single target echo. This requires that the beam function can be calculated everywhere where the transmit beam damping is less than $-10 \mathrm{~dB}$. If a table represents the calibrated beam function, the problem is to find the value of the beam damping in the table at the angles closest to those detected. If necessary, interpolations can be computed if the table has not enough resolution.

Most of the different plots of single echo Target Strength against observation aspect indicate that the detected Target Strength increases with aspect angle, in particular around and beyond $\theta_{-20}$. The increasing value of the weakest detected echoes with aspect angle is simply caused by the fact that many echoes far out in the beam are too week to be detected due to the beam damping. The increasing value of the strongest echoes with aspect angle has several causes. One is that the number of candidate echoes increases with aspect angle (see (7) and (8)), and it is likely that the strongest echo strength of many echoes is bigger than the strongest among fewer echoes closer to the acoustic axis. 
Also, positive errors in the detected phase angles of echoes lead to overcompensations of the beam damping, and this effect is stronger the steeper the beam function is, that is far out in the main lobe. Here, errors in detected phase angles are common. This is the same effect that is described in [4].

Lastly, in this paper, where beam compensation is computed from a beam model, the beam compensation will be wrong if the beam damping based on the model is different to that required for the actual beam. If the relation between the model and the actual beam function is as in Fig. (1), beam compensation far out in the main lobe is too strong. The author does not think that this effect is serious out to $\theta_{-20}$, but beyond $\theta_{-20}$ it is serious, according to data in this study.

The author of this paper hoped to develop a beam independent estimator of the Echo Value Constant by (10). However, (10) seems to have on average too high positive bias, and (12) that often behave better, is in some cases too low. An unfortunate property with (12) is that it depends on the number of echoes received within about $60 \%$ of the half power angle of the transducer. If this number happens to be too high or low, the corrections made further out in the main lobe will be wrong. The problem that estimator (12) depends on the parameter $s_{i}$ associated with undetected echoes is judged to be a minor problem. It may produce some nonnegligible bias in some situations with big variations in the sailing speed and/or systematic change in the ping-rate, but this happens seldom.

It may be possible to develop a more advanced estimator that depends on a better analysis of the number of echoes at different observation aspect angles. It is tempting to use the apparent true hypothesis that the correct single echo Target Strength and beam compensated integrated echo strength are independent of the observation aspect for small angles, that is within $\theta_{-20}$ for most transducers used in acoustic surveys. However, this is not easy to use when the purpose is to derive a "beam independent" estimator of the Echo Value Constant. A modified beam independent estimator should also preferably be based on the echo parameter values used to compute the estimator, and not use any general compensation. This is because there are many different situations that affect the number of echoes at different angles, such as depth or/and noise, mixture of different species including plankton, different fish densities, fish close to the bottom and so on. An improved estimator should be based on better knowledge about the different factors that affect the empirical distribution of observation aspect angles of single target echoes, and this will not be done here.

It may also be possible to develop a beam independent estimator of the Echo Value Constant based on model (1). Then (4) must be used to estimate both $a$ and $k$ by the method of least square. This has been looked into enough to conclude that it is mathematically and computationally possible, although it requires computed values of the derivative of the Bessel function, which is straightforward by using the power series of the derivative. This method has not been tried, because $k$ is expected to be estimated with negative bias, i. e. giving a wider beam than the actual. The reason for this is the positively biased fish back scattering strengths out in the main lobe. The author believes that it is difficult to compensate for this, because weighting down echoes out in the beam will at the same time reduce the information about $k$.

When using "in situ" - detected single fish echoes to convert "integrator values" to fish densities, there is always a problem with choosing a good single echo threshold. Most often the echo signal does contain echoes from other organisms than the target fish population, and these have to be filtered away. This problem is easy when the echo strengths from fish and the alien organisms do not overlap much. However, this happens seldom, and deciding a value for the single echo threshold is usually a compromise between accepting some alien echoes while rejecting some fish echoes. The threshold values used in this paper do not necessarily give the best estimates of the Echo Value Constants, but choosing the best threshold is not the topic of this paper.

The way of setting Integrator threshold in this paper is only applicable when the echo signal consists of mainly single target echoes. In particular, if the plankton echoes are heavily overlapping, it is not possible to filter them away by means of an Integrator threshold.

The average Target Strength values given in the tables show the effect of positive biased echo strengths of single echoes received at big observation angles in the main lobe. It shows that the weighted average are the smallest in almost all cases, and this is likely to be closest to the true value. However, the average Target Strength values indicate that the Target Strength bias is not dramatic within the beam angle at $-10 \mathrm{~dB}$ transmit beam damping. There are two effects on the Target Strength values given here that should be mentioned. The first is that Target Strength is not accurately calibrated in this work, and the values may therefore have a small, constant bias. This will, however, not affect the relative variation with observation aspect. The other effect is caused by the difference between the beam model and the actual beam. Beam compensations are computed from the beam model, but the bias caused by this is believed to be small for observation aspects within $-10 \mathrm{~dB}$ transmit beam damping.

\section{CONCLUSION}

Two types of estimators of the mean Echo Value Constant are adjusted to reduce the influence of echoes from the outer main lobe. They are tried and compared with real acoustic data. Detected single target echoes are subject to an increasing average positive bias of echo strengths with increasing observation angle.

One new estimator type, see (2), seems to be better than the old that seemed to have a moderate positive bias. This are estimator types that require a model of the beam dependent average integrated echo strength of fish as a function of observation angle.

The other type of estimators, see (6), is independent of a model of the beam dependent integrated echo strength, but requires a representative sample of single target fish echoes within the observation angle where the transmit beam has dropped $-10 \mathrm{~dB}$ in beam intensity. In general, it is not possi- 
ble to collect a representative sample of single target echoes within this angle interval, since many echoes from far out in the main lobe fails to be detected. Two different adjusted estimators, see (10) and (12), of this type to compensate for the loss of many echoes in the outer part of the main lobe are compared with real acoustic data. One of them seems to have a positive bias, although moderate in many cases. But this estimator don't compensate for the positive bias of detected echo strength. The other estimator was often better, but seemed to be unstable with negative bias in special cases. The acoustic data used represented many recordings with different depth distributions and fish densities.

Although the used single target echo detection routine integrates the detected echoes, it also detects the peak values of these echoes. Different average beam compensated peak values from within $-10 \mathrm{~dB}$ transmit beam damping are computed and converted to "in situ" Target Strength. It is demonstrated that weighted averages with low weigh of echoes received from the outer main lobe give lower mean Target Strength than that of an ordinary average of echoes. This holds in all except one case with high fish density. The difference was not dramatic, and was in most cases less than 1 $\mathrm{dB}$.

\section{REFERENCES}

[1] M. Aksland, "An alternative echo-integrating method", ICES Journal of Marine Science, vol. 62, pp. 226-235, 2005.
[2] R. E. Craig, "Redefinition of Sonar Theory in Terms of Energy", In: Selected papers of the ICES/FAO symposium on Fisheries Acoustics, Bergen, Norway, 21-24 June 1982". FAO Fisheries Report., No. 300 Nakken O. and Venema S. C., Eds., 1983.

[3] X. Zhao and E. Ona, "Estimation and compensation models for the shadowing effect in dense fish aggregations", ICES Journal of Marine Science, vol. 60, pp. 55-163, 2003.

[4] D. J. Fleischman and D. L. Burwen, "Correcting for positionrelated bias in estimates of the acoustic backscattering crosssection”, Aquatic Living Resources, vol. 13, pp. 283-290, 2000.

[5] M. J. Henderson, J. K. Horne and R. H. Towler, "The influence of beam position and swimming direction on fish target strength", ICES Journal of Marine Science, vol. 65, pp. 226-237, 2007.

[6] M. Aksland. "Applying an alternative method of echo-integration", ICES Journal of Marine Science, vol. 63, pp. 1438-1452, 2006.

[7] D. A. Demer, M. A. Soule and R. P. Hewitt, "A Multi-frequency method for improved accuracy and precision of in situ target strength measurements", Journal of Acoustical Society of America., vol. 105, pp. 2359-2376, 1999

[8] M. A. Soule, I. Hampton and M. Barange, "Potential improvements to current methods of recognizing single targets with a split-beam echo sounder", ICES Journal of Marine Science, vol. 53, pp. 237243, 1996.

[9] R. J. Urick. Principles of Underwater Sound, $3^{\text {rd }}$ ed., McGraw-Hill, New York, 1983, p. 423

[10] E. Bethke. "The evaluation of noise- and threshold-induced bias in the integration of single-fish echoes", ICES Journal of Marine Science: Journal du Conseil., vol. 61, pp. 405-415, 2004.

[11] P. Reynisson, "Evaluation of threshold-induced bias in the integration of single fish echoes", ICES Journal of Marine Science, vol. 53, pp. 345-350, 1996.

(c) Magnar Aksland; Licensee Bentham Open.

This is an open access article licensed under the terms of the Creative Commons Attribution Non-Commercial License (http://creativecommons.org/licenses/by-nc/3.0/) which permits unrestricted, non-commercial use, distribution and reproduction in any medium, provided the work is properly cited. 